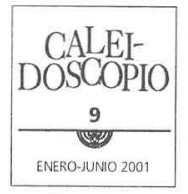

\title{
Migración internacional y crecimiento regional en el estado de Zacatecas
}

VOLKER HAMANN

Maestria en Ciencia Política/Universidad Autónoma de Zacatecas

INTRODUCCIÓN

El fenómeno de la migración de trabajadores internacionales ha aumentado en los últimos años, con sus consecuencias sociales y económicas, y será una cuestión de suma importancia en el nuevo siglo. Los movimientos migratorios se incrementaron en los últimos 30 años. En 1965, 75 millones de personas no vivían en sus países de origen, número que aumentó hasta 120 millones en 1990, lo cual equivale a un crecimiento promedio de $1.9 \%$ por año. Esto sin contar los movimientos internos, cuyo número es aún mayor. En 1981, solamente la India registró 200 millones de migrantes al interior del país, mientras que se estimó en 90 millones su emigración internacional para el mismo año. ${ }^{1}$

Según la economía neoclásica, la globalización, el libre comercio y el movimiento del capital deberían provocar una reducción de los movimientos migratorios, bajo el supuesto de que se disminuyen las diferencias en la productividad marginal entre los países. Sin embargo, las evidencias empíricas muestran lo contrario. Los movimientos migratorios ya existentes crecieron

1 Hania Zlotnik, "International Migation 1965-96: An Overview", Population and Development Review, 24 (3), 1998, pp. 429-4678. 
aún más. Obviamente, el fenómeno de las migraciones internacionales es muy complejo y no puede ser explicado solamente por diferencias de ingresos o por inversiones extranjeras directas. Por el contrario, la migración se puede considerar como un símbolo de la globalización, ya que la destrucción de las estructuras económicas y sociales tradicionales, por medio de la globalización, muchas veces favorece los movimientos migratorios. $^{2}$

Las políticas migratorias de los países receptores y expulsores, en la mayoría de los casos son motivo de acaloradas controversias. Algunos de los debates se enfocan en cómo integrar a los migrantes en la sociedad receptora o cómo reducir los flujos de inmigrantes,${ }^{3}$ ignorando que las regiones de origen de los migrantes también se ven afectadas por estos movimientos, por lo cual las políticas referentes a esta cuestión son casi inexistentes. Es importante, por ello, examinar también las consecuencias económicas y sociales de la migración en las regiones de origen, para así poder construir una base científica que ayude a encontrar políticas complementarias.

2 David P Lindstrom, "Economic Opportunity in Mexico and Return Migration from the United States", Demography; Volume 33. Number 3. August, 1996, pp. 357-374; Hélène Pellerin, New Global Migration Dynamics. Stephen Gill (Ed.), Globalisation, Democratisation and Multilateralism, 1997, pp. 105-125; Richard C Jones, "Introduction: The Renewed Role pof Remittances in the New World Order", Economic Geography: 74, 1998, pp. 1-7; Christof Parnreiter, "Migration: Symbol, Folge und Triebkraft von globaler Integration”, Erfahrungen aus Zentralamerika; Christof Parnreiter, Andreas Novy, Karin Fischer, "Globalisierung und Peripherie. Umstrukturierung in Lateinamerika, Afrika und Asien", Historische Sozialkunde, 14 ed. Frankfurt am Main, Wien: Brandes \& Apsel, Südwind, pp. 129-149.

3 David S Bell \& John Gaffney, European Immigration Policy. Sami Mair, Contemporary European Affairs, Oxford, 1990; Daniel Kubat, The Politics of Migration policies. Settlement and Integration. The first World into the 1990s, 2nd Edition, New York, 1993. 
Por sus raíces históricas, la migración frecuentemente responde a fenómenos regionales específicos. Jones sostiene que las diferentes regiones de México, aun presentando las mismas bases económicas, no muestran los mismos patrones migratorios. También afirma que regiones con los mismos patrones migratorios pueden diferir mucho respecto a su nivel de desarrollo económico. ${ }^{4}$ Estas diferencias interregionales no han recibido mucha atención científica; por esto, en el presente texto se trata de analizar parte del material existente producto de diferentes autores, para finalmente sugerir algunas áreas de interés para investigaciones posteriores.

En las líneas que siguen vamos a examinar el fenómeno migratorio en un contexto histórico hasta llegar a los patrones migratorios actuales, destacando los efectos que el TLCAN puede tener en las economías y los pronósticos que se han hecho para el futuro de la migración. Más adelante trataremos de la conexión entre migración y crecimiento económico, destacando la importancia de las remesas de los trabajadores migrantes y su impacto en el desarrollo regional. Enfocando los volúmenes de capital, su uso y sus implicaciones económicas. Finalmente daremos un resumen de los resultados y aportaremos algunas conclusiones.

\section{Migración entre México y los Estados Unidos de América}

Los movimientos migratorios entre México y los Estados Unidos constituyen uno de los flujos más grandes en el mundo. De 1970 a 1990 la población en el vecino país del norte de origen mexicano creció de 760,000 a 4.3 millones, lo cual equivale a una taza de crecimiento anual promedio de $8.7 \%$. Según ciertas estimaciones, el número aumentó a 6.7 millones en 1996, lo que hace a la migra-

4 Richard C Jones, Ambivalent journey: U.S. migration and economic mobility in north central Mexico. Richard C. Jones.-Tucson : Univ. of Arizona Press, 1995. 
ción entre México y los Estados Unidos única en el mundo, en el sentido de que casi todos los migrantes mexicanos (97\%) tiene como destino los EUA, incrementándose con esto los lazos económicos, sociales y culturales entre los dos países. ${ }^{5}$ Por lo anterior, es evidente que el fenómeno de la migración internacional de fuerza de trabajo es de vital importancia para México, lo que ha llamado la atención de una parte importante de la comunidad científica. ${ }^{6}$

En una aproximación al fenómeno de los movimientos migratorios entre México y los EUA, sobresale la frontera con más de $3,000 \mathrm{kms}$. También hay que tomar en cuenta que una gran parte del suroeste de los EuA perteneció a México hasta la primera mitad del siglo XIX.

La consolidación de los latifundios y la modernización de la agricultura mexicana durante el periodo de Porfirio Díaz (18761911) creó el fundamento no sólo para la Revolución Mexicana, sino también concentró un potencial enorme de gente que se liberó de las ataduras de sujeción y que estaba dispuesto a emigrar para mejorar sus condiciones de vida. Desde el inicio de la Revo-

5 Hania Zlotnik, op. cit.

6 Secretaría de Relaciones Exteriores; Commission on Immigration Reform (Ed.). Estudio Binacional México-Estados Unidos sobre Migración, México, 1997; , Sherman Robinson et al, "Agricultural Policies and Migration in a U.S.-Mexico Free Trade Area: A Computable Ceneral Equilibrium Análisis", Journal of Policy Modeling, 15 (5\&6), 1993, pp. 673-701; J. Edward Taylor, Micro Economy-Wide Models for Migration and Policy Analysis: an Application to Rural Mexico, Paris, OECD, 1995; J. Edward Taylor et al. Agricultural Policy Reforms and Village Economies: A CGE Analysis from Mexico. Journal of Policy Modeling, 1996; Demetrios C. Papademetriou, Illegal Mexican Migration in the United Stales and US Responses. International Migration, 1993, pp. 314-335; Carlos Carrocho, Cambios en la Estructura funcional del Sistema Migratorio Mexicano; Adrián Guillermo Aguilar, Desarrollo Regional y Urbano. Tendencias y Alternativas, tomo II; José Luis Calva, Seminario Nacional sobre Alternalivas para la Economía Mexicana, México, D.F., 1995. 
lución hasta los años treinta, muchos emigraron hacia los EUA, huyendo de la situación política y económica inestable. Así también, durante la política de industrialización mediante la sustitución de importaciones, que se mantuvo de 1940 a 1982, aumentó el número de migrantes potenciales. Debido a que los agricultores de pequeña propiedad privada y ejidatarios no recibieron los soportes económicos adecuados, en cambio el sector industrial recibió muchos subsidios. Con la crisis de los ochenta y los programas de ajuste presupuestal se aumentó, aun más, el número de personas dispuestas a emigrar.

Debemos considerar que en el otro lado de la frontera en varias épocas hubo gran demanda de fuerza de trabajo barata. En los años ochenta del siglo XIX, las compañías de ferrocarriles estadounidenses reclutaron trabajadores mexicanos para la construcción de las vías ferroviarias. Las contrataciones se realizaron en algunos de los estados que ahora muestran las migraciones más altas. La crisis económica de los años treinta causó la deportación de muchos migrantes y de ciudadanos norteamericanos con origen mexicano. Sin embargo, como consecuencia del ingreso de los Estados Unidos a la Segunda Guerra Mundial, en 1942, se inició el "Programa Bracero" bajo el cual fueron contratados gran número de trabajadores mexicanos, indispensables para hacer funcionar la industria de guerra de los EuA. Después de que el "Programa Bracero" fue liquidado en 1964, la agricultura norteamericana continuó demandando fuerza de trabajo barata por temporadas, lo que motivo un incremento de la inmigración ilegal.

Como consecuencia de las distintas causas históricas, los movimientos no están distribuidos homogéneamente en toda la república. La mayoría de los migrantes provienen de cinco estados del centro del país: Guanajuato, Jalisco, Zacatecas, Durango y Michoacán. Este último tiene los números absolutos más altos de migrantes, mientras que Zacatecas tiene el más alto porcentaje de migrantes en relación con su población. En el pasado reciente otros estados se han agregado a la lista, Baja California, 
Chihuahua, Guerrero, Estado de México y el Distrito Federal. ${ }^{7}$ En estas entidades la migración se diferencia mucho internamente entre los diferentes municipios. La presión sobre la economía mexicana por los programas de ajuste y el TLCAN, obviamente, no provoca las mismas reacciones en todas las regiones, aunque las condiciones se parezcan; por eso, el impacto de la migración internacional establece diferencias regionales a considerar. ${ }^{8}$

Una mirada más cercana a las diferencias regionales establecidas por los movimientos migratorios muestra que, de los 2,428 municipios de la república el $62 \%$ tienen movimientos de migración, $43 \%$ nada más muestran una actividad migratoria baja y $18 \%$ una actividad muy baja. En los estados del sureste del país (Chiapas, Tabasco, Campeche, Yucatán, Quintana Roo), 66.5\% de todos los municipios no tienen movimientos migratorios, mientras en los estados del noroeste casi todos los municipios toman parte en las migraciones y solamente 4.5\% (109) del total de los municipios muestran una actividad migratoria muy alta. De éstos, el $80 \%$ son municipios con una población menor a 20,000 habitantes. Obviamente, el fenómeno de la migración es más grande en las áreas rurales. El 88\% de estos 109 municipios están ubicados dentro de los nueve estados con mayor movimiento migratorio: Jalisco, Michoacán, Guanajuato, Zacatecas, Durango, Chihuahua, San Luis Potosí, Guerrero y Oaxaca. Si tomamos en cuenta que casi todos los municipios urbanos muestran cierta

7 Sin considerar la zona metropolitana (Estado de México y Distrito Federal), la cual apenas en los últimos años se ha vuelto zona de emigración), y Guadalajara, advertimos que la mayoría de los migrantes provienen de zonas rurales, en las que vive sólo el $20 \%$ de la población nacional.

8 Gustavo López Castro y Sergio Zendejas Romero, Migraciones Internacionales y sus Efectos Regionalistas. Adrián Cuillermo Aguilar, Desarrollo Regional y Urbano. Tendencias y Allernativas, Tomo Ir. José Luis Calva, Seminario Nacional sobre Alternativas para la Economía Mexicana, México, 1995. 
actividad migratoria, la migración rural domina por sobre la migración urbana. ${ }^{9}$

El carácter regional de los movimientos migratorios en México es aún más extremo por las grandes diferencias existentes en la economía mexicana. Por un lado, el sector industrial está compitiendo con las economías más avanzadas del mundo; por otro, unos sectores muestran un perfil que no se distingue de los países más pobres y menos desarrollados. Este dualismo también se refleja en la distribución de los ingresos. Dentro del mundo occidental, México -después de Brasil- tiene las diferencias más grandes entre los ricos y los pobres. ${ }^{10}$ En México, los ingresos y el PIB varían mucho por estado. La zona metropolitana de la Ciudad de México por ejemplo, genera alrededor de $40 \%$ del PIB nacional y concentra gran parte de las inversiones públicas, acrecentando así, aun más, los desequilibrios existentes. ${ }^{11}$ Otro ejemplo de este desequilibro se percibe en el PIB por persona. En los estados de Nuevo León, Campeche y Quintana Roo es tres o hasta cuatro veces más alto que en los estados de Chiapas, Oaxaca y Zacatecas. ${ }^{12}$ Los emigrantes, que en su mayoría provienen de las zonas rurales, obviamente no tienen acceso a los trabajos mejor pagados del avanzado sector industrial.

En lo que toca a Zacatecas, desde la última década del siglo XIX aporta grandes cantidades de trabajadores para la economía norteamericana; en consecuencia, la tasa de crecimiento poblacional siempre ha estado abajo del promedio nacional, mien-

9 Gustavo Verduzco y Kurt Unger, The Impact of Migration on Economic development in Mexico, OECD, 1998.

10 Jorge A Bustamante, Nafia and Labour Migration to the United States. Victor Bulmer-Thomas, Nikki Craske \& Mónica Serrano (eds.) México and the North American Free Trade Agreement, 1994.

11 Leonardo Curzio Gutiérrez, "El Desarollo Regional frente al Proceso de Integración Económica", Desarrollo Regional y Urbano. Tendencias y Alternativas, 1995, pp. 133-144.

12 INEGI: www.inegi.gob.mx 
tras que las tasas de natalidad y la mortalidad en el estado son casi iguales al promedio de la República Mexicana. En 29 de los 56 municipios de la entidad, el crecimiento poblacional absoluto fue negativo durante los años noventa. ${ }^{13}$

La migración de una persona es más probable si la familia o por lo menos la comunidad tiene alguna experiencia migratoria, porque estos conocimientos reducen los riesgos y problemas en el cruce de la frontera. Es decir, este capital social reduce los costos de la migración. Conviene apuntar que estas redes sociales o redes migratorias son mucho más fuertes en las áreas rurales que en las urbanas. ${ }^{14}$ También, la existencia de recursos monetarios hace más probable la migración, porque se le hace más fácil al migrante pagar los gastos, como el viaje y el "coyote". La posesión de una casa o un pequeño negocio, en cambio, reduce la probabilidad de migración, porque la necesidad de ingresos no es tan alta como en las familias sin propiedades. La posesión de terrenos, en cambio, incrementa la probabilidad de migrar, un hecho que puede ser interpretado como, la necesidad de capital adicional para poder trabajar bien y eficazmente las tierras.

La economía neoclásica propone la reducción de los movimientos migratorios a través de salarios más altos y un desarrollo industrial. Sin embargo, hay evidencia de que en algunas comunidades rurales con un desarrollo económico positivo las tasas de migración son extremadamente altas. Dentro de la complejidad que presenta la migración, es posible sostener que la probabilidad de migrar es más alta en comunidades rurales que en las urbanas. ${ }^{15}$ Esto no es suficiente para resolver la cuestión de si el

13 Miguel Moctezuma Longoria, Redes Sociales, Comunidades filiales y clubes de migrantes, tesis de doctorado, 1999.

14 Idem.

15 Douglas S. Massey, Kristin E. Espinosa, "What's driving Mexico-U.S. Migration? A Theoretical, Empirical, and Policy Analysis", American Journal of Sociology, Volume 102, Number 4, January, 1997. 
crecimiento económico es un factor que interviene en el proceso de migración, o al revés. Es un hecho sorprendente que la mayoría de los migrantes han tenido ingresos antes de migrar a los EUA, pero la mayoría considerara insuficientes sus ingresos en México. ${ }^{16}$

La globalización de la producción y las transacciones financieras representan un reto en los gobiernos nacionales para mantener el control de sus economías. Esta globalización también tiene grandes impactos in la migración internacional de fuerza de trabajo, lo que involucra a los países de origen y de recepción. ${ }^{17}$ Aunque el problema de la migración ya era obvio y predecible, éste no fue un tema de discusión en las negociaciones del TLCAN. ${ }^{18}$

El TLCAN incluye la reducción de los subsidios agrícolas en México durante un periodo de 10 años. Así, las bases económicas para los pequeños propietarios, que antes de 1994 dependieron de la ayuda del gobierno, están en peligro. Debido al bajo rendimiento de los productos agrícolas y al deficiente grado de tecnificación, para la mayoría de los pequeños productores ya no será posible vender sus productos a precios competitivos. Se supone que gran parte de los miembros de estos hogares van a buscar una solución migrando al norte. Algunas cifras estiman que unos 600,000 campesinos emigran a los EuA. ${ }^{19}$ Por otro lado, los agricultores mexicanos ya han diversificado sus fuentes de ingresos durante los últimos años; por eso, la dependencia del maíz y del frijol se ha reducido, mientras la parte de los ingresos que proviene de la migración internacional se ha incrementado en los

16 Wayne A. Cornelius, Philip L. Martin, "The Uncertain Connection: Free Trade and Rural Mexican Migration to the United States", International Migration Review, 1993, pp. 484-512.

17 Nora Hamilton, Norma Stoltz Chinchilla, "Global Economic Restructuring and International Migration: Some observations based on the Mexican and Central American experience", International Migration, 1996, pp. 195-227.

18 Bustamante, op. cit.

19 Cornelius, op. cit. 
años recientes. Esta estrategia de la diversificación de los ingresos sólo redujo parcialmente la dependencia, pues en general podemos considerar que solamente ha cambiado la forma en que se da la dependencia. Y en el futuro van a depender aún más de las remesas de los migrantes.

La integración económica entre México y los EUA a través del TLCAN no sólo causó un aumento en los movimientos migratorios entre los dos países, sino también una gran influencia en los flujos migratorios internos en México. Éstos se redirigieron hacia las industrias maquiladoras, que en su mayoría están ubicadas en la zona fronteriza entre los dos países. ${ }^{20} \mathrm{La}$ industria maquiladora, ubicada en las partes poco pobladas del país, creció de 113,897 trabajadores y empleados en 1980 a 1,067,217 en 1999. ${ }^{21}$ La mayoría de los trabajadores se mudó desde otras partes de la República hacia el norte. Para unos, esta migración interna representó nada más el primer paso para después cruzar la frontera hacia los EUA.

Con o sin libre comercio no será posible en los próximos años reducir la presión migratoria de México hacia los EUA. Las diferencias en las oportunidades de trabajo y los salarios son demasiado grandes para que puedan ser equilibrados en el corto plazo, y la existente integración económica, social y cultural -es decir, las redes sociales- van a facilitar la migración en el futuro.

El salario mínimo del estado de Zacatecas, por ejemplo, corresponde a 4 dólares por día, una suma que en los EUA se puede ganar fácilmente en una hora. El intento de reducir la migración zacatecana a través del establecimiento de plantas maquiladoras en el estado fracasó, porque los salarios no pueden competir con los ofrecidos a los zacatecanos en los EUA. Las redes migratorias que existen han disminuido los riesgos y costos de la migración, así que para mucha gente es más fácil encontrar un trabajo en los

\footnotetext{
20 Zlotnik, op. cil.

21 INEGI: www.inegi.gob.mx
} 
EUA que en Zacatecas o en cualquier otro estado de la República. Proponer que a largo plazo los movimientos migratorios nada más pueden ser frenados por un crecimiento y desarrollo económico en las regiones de origen, no es suficiente ya que no hay alternativa a un crecimiento inducido por el comercio ${ }^{22}$. Esto es, en los primeros años la inversión extranjera directa y el crecimiento de las empresas exportadoras no tienen gran influencia en los movimientos migratorios, porque los migrantes no tienen las habilidades que se requieren para estos nuevos trabajos. Por eso, para el lado mexicano también se necesita un ajuste en la educación, que cumpla con los perfiles requeridos de las nuevas industrias. ${ }^{23}$ Por tanto, se puede esperar que las nuevas inversiones se sigan realizando en las regiones que ya muestran tasas de crecimiento altas, de manera que las regiones rurales van a participar en el crecimiento de manera marginal.

Como consecuencia de la reestructuración global y los cambios políticos, los movimientos migratorios están sometidos a cambios, los cuales no forzan a repensar los conceptos ortodoxos de la migración. En el pasado, los movimientos migratorios se podían dividir en tres categorías:

- Emigración/ Inmigración (Integración)

- Migración temporal (sin integración, regreso seguro al país de origen)

- Diáspora (formación de minorías étnicas, sin integración cultural o social)

Los nuevos patrones de la migración internacional de fuerza de trabajo muestran que estos conceptos necesitan ser repensados. En muchas áreas un nuevo patrón migratorio se establece: la transmigración, que ha sido posible en los tiempos de globalización y por las nuevas tecnologías de transporte. En contraste con los

22 Corneluis, op. cit.

23 Bustamante, op. cit. 
patrones migratorios mencionados, los transmigrantes no pertenecen a un cierto ambiente cultural o social, trabajan y viven alternando en el país de origen y en el de destino. ${ }^{24}$ Mientras que en el pasado el patrón migratorio dominante entre México y los EUA era la migración laboral temporal, ahora los transmigrantes ganan significatividad. Esto causa cambios en el sistema cultural y social tanto en las regiones de origen como en las de destino. Más y más migrantes se establecen en los EUA y el contacto con las regiones de origen se reduce. ${ }^{25}$ Esto ha sido posible especialmente por un cambio de la política de inmigración de los EUA. Con la aprobación de IRCA (Acta para la Reforma y el Control de la Inmigración) en 1986, muchos migrantes que antes no tenían un estatus legal obtuvieron la oportunidad de legalizarse. Además, el IRCA abrió las puertas a la reunificación familiar; es decir, un migrante documentado puede llevar a toda su familia a los EỤA. Así, el número de migrantes documentados aumentó y muchos tomaron la oportunidad para establecerse en aquel país.

\section{Migración, Remesas y Crecimiento Económico}

Aunque hay muchas investigaciones en esta área, las opiniones acerca de las interdependencias entre migración y desarrollo en países exportadores de fuerza de trabajo todavía varían mucho. Unos investigadores concluyen que la migración laboral no favorece el crecimiento económico en los países exportadores de mano de obra, considerándola hasta dañina, ${ }^{26}$ mientras que otros su-

21 Ludger Pries, “Transmigranten' als Typ von Arbeitswanderern in plurilokalen sozialen Räumen. Das Beispiel der Arbeitskräftewanderung zwischen Puebla/Mexiko und New York". Soziale Welı.; Jahrgang 49, Heft 2, 1998 , pp. 135-149.

25 Fernando Lozano Ascencio, "Remesas: ¿fuente inagotable de divisas?" Ciudades, 35, julio-septiembre, 1997, pp. 12-18.

26 Cünter Schiller, "Die Auswanderung von Arbeitskrüften als Problem der wirtschaftlichen Entwicklung”, Das Argument, 1971, pp. 800-809; M. G. 
gieren una vinculación positiva entre migración y desarrollo. ${ }^{27} \mathrm{Si}$ embargo, todos concluyen que las remesas de los migrantes toman un rol importante en el vínculo entre migración y desarrollo. ${ }^{28}$

México pertenece a los cinco países en el mundo que más remesas reciben, pero hasta ahora no ha implementado un sistema que permita canalizar las remesas directamente a opciones productivas, como lo han hecho Corea del Sur, Tailandia e

Quibria. "On generalizing the economic analysis of international migration: a note", Canadian Journal of Economics, XXI. No. 4. November, 1988, pp. 874-876; Wong, Kar-Yiu, "The economic analysis of international migration: a generalization", Canadian Journal of Economics, XIX. No. 2. 1986, pp. 357-362; H. Rubenstein, "Migration, Development and Remittances in Rural Mexico" International Migration, 1992, pp. 127-151.

27 Reginald Appleyard, "International Migration and Development -An Unsolved Relationship", International Migration, 1992, pp. 251-265; Frank Kirwan, "Migration and Emigrants" Remittances: Theory and Evidence from the Middle East", Mats Lundahl (Ed.), The Primary Sector in Economic Development: Proceedings from the Seventh Arne Ryde Symposium, 1995, pp. 253-270; Sharon Stanton Russell, "Remittances from International Migration: A Review in Perspective”, World Developmeni. 14, No. 6, 1986, pp. 677-696; Sharon Stanton Russell, "Migrant Remittances and Development”, International Migration, 1992, pp. 267-287; Charles W. Stahl \& Fred Arnold, "Overseas Workers' Remittances in Asian Development”, International Migration Review, XX. No.1. 1986, pp. 899925; Charles W Stahl \& Ahsanul Habib, “The Impact of Overseas Workers. Remittances on Indigenous Industries: Evidence from Bangladesh", The Developing Economies, 1989, pp. 269-285.

Lozano, op. cil.; Jorge Durand \& Patricia Arias, "Las remesas: ¿continiudad o cambio?” Ciudades, 35, julio-septiembre, 1997, pp. 3-11; Fred Arnold, "The Contribution of Remittances to Economic and Social Development", Mary Kritz, (Ed.). International Migration Systems: a Clobal Approach, 1992, pp. 205-220; Dennis Conway \& Jeffrey H. Cohen, "Consequences of Migration and Remittances for Mexican Transnational Communities", Economic Geography, No. 74, 1998, pp. 26-44; Phillip Martin, "Trade, Aid and Migration", International Migration Review, 26, Spring, 1992, pp. 162 172; Agustín Escobar Latapí, "Low Skill Emigration from Mexico to the United States. Current Situation and Government Policy", International Migration, Vol. 37 (1), S. 1999, pp. 153-182. 
Indonesia, desde ya hace mucho tiempo. ${ }^{29}$ Es importante recordar que las remesas sólo pueden estimular el desarrollo si su monto excede el valor de la producción que los migrantes hubieran generado quedándose en su lugar de origen. ${ }^{30}$ Tomando el caso de México y Zacatecas, podemos estar seguros de que esto es así.

En su análisis, Rivera-Batiz ${ }^{31}$ examina los impactos de la migración en la estructura económica de un país exportador de mano de obra, concluyendo que el incremento de los precios de los bienes no comerciables afecta negativamente el bienestar de los no-migrantes. En sus encuestas sobre el nivel de desarrollo de un país, Knerr ${ }^{32}$ descubre que el impacto de la migración de fuerza de trabajo tiene efectos similares al incremento de las exportaciones del sector primario. El incremento de los flujos de divisas hacia el país provoca un aumento de las importaciones y con esto se crea una tendencia a la desindustrialización, también conocida como el "Dutch Disease" o la enfermedad holandesa. Como consecuencia, la región o el país se especializan en la producción de fuerza de trabajo migrante, un fenómeno que ya se ha observado también en el caso de Zacatecas, ${ }^{33}$ provocando aumentos en

29 Athukorla, Premachandra. "Improving the Contribution of Migrant Remittances to Development: The Experience of Asian Labour-exporting Countries”. En International Migralion. Núm. 31, 1993, pp. 103-122.

30 Taylor, op. cit.

31 Francisco L. Rivera-Bátiz, "International n.igration, non-traded goods and economic welfare in the source country", Journal of Development Economics, 11, 1982, pp. 81-90; Francisco L. Rivera-Bátiz, "International migration, non-traded goods and economic welfare in a two-class economy", Journal of Development Economics, 16, 1984, pp. 325-330.

32 Beatrice Knerr, "Labour Emigration and its effects on the Economies of South Asia", Internationeles Asienforum (International Quaterly for Asian Studies), Vol. 20. No. 3-4, 1989, pp. 263-293; Beatrice Knerr, "Labour export from South Asia: another Case of the Dutch Disease?", Ciorgio Borsa (ed.). Trade and Politics in the Indian Ocean; Historical and Contemporary Perspecives, 1990, pp. 169-221.

33 Raúl Delgado Wise el al. Para enlender a Zacalecas, Zacatecas, 1994. 
toda clase de bienes y servicios que se demandan a partir de las remesas. Sin embargo, si los bienes comerciables son importados, la estructura de la producción cambia hacia la producción de bienes no comerciables. Igualmente, si en una región los factores de producción tienden a salir, esto repercute en un incremento en los precios de los bienes no comerciables, lo cual reduce también la competitividad de la región. Estos argumentos están respaldados por Cabrales Barajas, ${ }^{34}$ quien ha descubierto que en regiones que reciben muchas remesas, los precios de terrenos y construcción aumentan, mientras la productividad en la agricultura se reduce.

Por otro lado, varios investigadores presentan evidencias de que las migraciones son parte de las decisiones familiares en busca de aumentar los ingresos. Bajo este supuesto, las remesas de los migrantes juegan un rol importante en el crecimiento de las regiones de origen, porque permiten aliviar las restricciones en la producción y las inversiones. ${ }^{35}$

En comparación con otras transferencias monetarias, como la ayuda para el desarrollo o programas de ajuste interregional, las remesas no se canalizan por las burocracias o mecanismos que permitan que los envíos puedan llegar directamente a los que menos ingresos tienen. Las remesas no son caridad y su distribución no esta conectada a ciertas reglas. Se trata de dinero ganado por la familia migrante y representa una fuente de ingresos de la que pueden estar orgullosos. ${ }^{36}$

La información acerca del monto y del uso de las remesas es de gran importancia cuando se estudia el vínculo entre migración y crecimiento. Históricamente, la transferencia de tecnología y

Luis Felipe Cabrales Barajas, "El refugio silencioso del ahorro migrante", Ciudades, 35, julio-septiembre, 1997, pp. 19-25.

Jorge Durand et al., "Migradollars and Development: A Reconsideration of the Mexican Case", International Migration Review, xxx, No. 2, 1996, pp. 423-444. 
de conocimientos de los EUA a México no ha tenido mucha importancia, lo que se refleja en las diferencias en el nivel de educación de ambos países. La mayoría de los migrantes trabaja en puestos que no requieren mucha preparación y casi no adquieren conocimientos que puedan utilizar productivamente en México. Zahniser y Greenwood ${ }^{37}$ hacen una comparación entre los migrantes de retorno y los años de educación en México, considerando la experiencia de trabajo en los EUA. Independientemente de cualquier apología, es significativo señalar los efectos positivos de una experiencia en los EUA, que en términos económicos es alrededor de dos veces superior a los ingresos que se pueden lograr por el efecto de 12 años de estudios en México. Con toda seguridad, éste es un elemento que interviene fuertemente para migrar. Por otro lado, hay que tomar en cuenta que los migrantes de retorno tienen que encontrar trabajo en México, lo cual muchas veces es difícil en las comunidades rurales. También es pertinente considerar que los migrantes que encuentran un trabajo bien pagado se queden en los EUA.

Entre los pocos ejemplos de transferencia de tecnología figuran las huertas de duraznos, en el municipio de Jerez. Por sus redes sociales particulares, grandes cantidades de migrantes de la comunidad Los Haro, en Jerez, se van a trabajar en las plantaciones de uva y durazno, en el valle de Napa, en California. Alrededor de 1968 los primeros duraznos fueron plantados en Jerez y desde entonces la región se volvió uno de los productores más importantes de durazno en México, aunque la carencia de capital ha impedido la modernización de los plantíos.

Existen varios estudios sobre el monto de dinero que se manda anualmente. Los cálculos están entre 5 y 6 billones de dólares

37 Steven S Zahniser \& Michael J. Creenwood. "Transferability of Skills and the Economic Rewards to U.S. Employment for Return Migrants in Mexico", Migralion between Mexico and the United Stales. Binational Study, Volume 3, 1998, pp. 1133- 1152 . 
por año. Para el periodo de 1980 a 1995, el Banco Mundial reportó un incremento de las remesas de 690 millones a 3.67 billones de dólares, lo cual equivale a una tasa de crecimiento de casi $11 \%$ anual. Sólo el valor total de las remesas representa el $1.5 \%$ o $2 \%$ del PIB mexicano, pero el impacto está concentrado en las áreas de alta migración. ${ }^{38} \mathrm{El}$ estado de Zacatecas, por ejemplo, recibe alrededor de un millón de dólares diarios. ${ }^{39}$ Los cálculos del monto exacto de las remesas son inexactos, porque no todas las transferencias se procesan por los canales oficiales. Grandes sumas llegan al país por las llamadas transferencias de bolsa, es decir, por migrantes que visitan sus familiares y dentro de sobres traen dinero en efectivo para ellos, vecinos y conocidos.

Encuestas revelaron que el $47 \%$ de todos los migrantes mandan remesas y ahorran dinero, el $22 \%$ sólo envían remesas, el $13 \%$ únicamente ahorra y $18 \%$ no hacen algo de lo anterior. La probabilidad de mandar remesas es mucho más alta si el migrante proviene de una región que muestra un desarrollo económico positivo. ${ }^{40}$ Como ya habíamos dicho antes, los movimientos migratorios se diferencian por regiones. Por eso la distribución en el país de las remesas es muy desigual, presentando ciertas concentraciones regionales. Sólo 10 de los 32 estados de la República Mexicana recibieron más del $77 \%$ de todas las remesas en $1991^{41}$; los EUA dominan como fuente de remesas, en segundo lugar, a muy larga distancia, está Canadá, con un $3 \%$ de las remesas. ${ }^{42}$

Los nuevos patrones migratorios también influyen en el monto de dinero que se manda, ya que cada día más y más migrantes se

38 Jorge Durand, William Kandel, Emilio A. Parrado \& Douglas S. Massey, "International Migration and Development in Mexican.Communities", Demography, Volume 33. Number 2, May, 1996, pp. 249-264.

39 Moctezuma, op. cil.

40 Durand el al. "Migradollars and Development", op. cit.

41 Michoacán, D.F., Estado de México, Jalisco, Aguascalientes, Guanajuato, Guerrero, Zacatecas, Chihuahua y Baja California. Cfr. Lozano, op. cit.

12 Lozano citado por López Castro y Zendejas Romero, op. cit. 
establecen en los EUA. Ahí encuentran su centro de vida y por eso se reducen los lazos culturales con las comunidades de origen. Como consecuencia de esto, el monto de las remesas se reduce también. Los migrantes mexicanos que tienen la residencia permanente tienen mayores ingresos, pero mandan menos dinero. Este movimiento hasta ahora está compensado por el aumento del número de migrantes. ${ }^{43}$ En el futuro el monto total puede disminuir con resultados desastrosos para las regiones de origen. En los años 70 y 80 los gobernadores Pámanes Escobedo (19741980) y Cervantes Corona (1980-1986) no lograron promover la industrialización del estado de Zacatecas, estableciendo sólo alguna infraestructura. La participación del sector industrial cayó de $5.4 \%$ en 1975 a $4.02 \%$ en 1985. Fue el gobernador Genaro Borrego (1986-1992) quien finalmente logró un crecimiento industrial más rápido. En 1991 se establecieron 16 industrias pequeñas, 7 medianas y dos grandes, creando un total de 2,376 empleos. Además, estaban en construcción 3 pequeñas industrias y 5 grandes, con posibilidades de crear alrededor de 9,500 empleos. ${ }^{44}$ En el año 1996 el porcentaje de participación de la industria manufacturera en el PIB subió al 5.1\%, pero todavía no alcanzó los niveles de 1975. ${ }^{45}$ La participación de la agricultura en Zacatecas era de $25.14 \%$, cuando a nivel nacional era apenas de 6.47\%. También la industria minera en Zacatecas estaba sobrerepresentada, con el $3.98 \%$, frente al $1.47 \%$ del PIB a nivel nacional. ${ }^{46}$ Esto muestra que "Zacatecas no ha logrado deshacerse de su especialización en la producción de bienes primarios, pese a los importantes avances

Lozano, op. cil. Jorge Santribañez Romellón, "Migration Flows Between Mexico and the United States", Trends in International Migration, edited by Max J. Castro, North-South Center Press, University of Miami, 1999.

José Manuel Martín Ornelas, Tenıativas de industrialización en los ıres úlıimos periodos gubernamentales en Zacatecas 1974-1992, Zacatecas, 1993.

45 INEGI: www.inegi.gob.mx

46 INECI: www.inegi.gob.mx 
del siglo xx" y conviene aclarar que las tendencias, lejos de revertir esta orientación económica, apuntan ha reforzarla." ${ }^{\text {47 }}$ Esto quiere decir que, además de la producción de bienes primarios, el estado de Zacatecas se ha especializado en la exportación de fuerza de trabajo, ${ }^{48}$ lo que con el tiempo podrá traer los mismos problemas que la dependencia de la extracción de minerales o de los productos agrícolas.

En el área de la infraestructura se puede observar un ligero avance. De 1991 a 1998 la red de carreteras pavimentadas en el estado de Zacatecas creció de 2,600 km a 2,778. Las actividades en el Aeropuerto Internacional de Zacatecas General Leobardo C. Ruiz, se incrementaron de un total de 7,351 vuelos en el año de 1992 a 7,404 vuelos en 1998. El número de pasajeros aumentó de 271,441 en 1992 a 323,278 en 1998. Otro resultado de la alta actividad migratoria en ciertos municipios de la entidad es que en el municipio de Jerez, que cuenta con 56,180 habitantes, existen 12 agencias de viajes, mientras que en la capital del estado, con 118,742 habitantes, nada más existen 11. En el municipio de Fresnillo hay 7 de estos negocios. Es obvio que los movimientos migratorios favorecen el establecimiento de agencias de viajes y que la estructura económica de los municipios más afectados cambia en este sentido.

Ciertamente, la superficie sembrada aumentó de 1,202,968 hectáreas en 1991 a 1,295,944 hectáreas en 1998. Pero hay que tomar en cuenta que el nivel de los salarios se redujo en los últimos años. Por ejemplo, las remuneraciones reales del personal ocupado en los establecimientos comerciales de la ciudad de Zacatecas bajaron. Estimaciones hechas a partir de 1994 (100\%)

47 Víctor M. Figueroa Sepúlveda, "Zacatecas", en Marginación y Pobreza en México, Ariel, 1995, р. 377.

18 Delgado Wise el al., op. cil. 
mostraron una reducción al 66.1\% para el área del mayoreo y al 76.3\% para el área del menudeo en el año de $1998 .{ }^{49}$

Hablando de los vínculos entre migración, remesas y crecimiento económico, la investigación no sólo se debería enfocar en el monto, sino también en el uso de los dólares que se mandan. El crecimiento económico de una región depende en gran parte del modo en que se gasten las remesas. Obviamente, la inversión tiene otras consecuencias además del consumo. Una encuesta en 22 comunidades de cuatro estados (Jalisco, Guanajuato, Michoacán y Nayarit), indica que únicamente $6.5 \%$ de las remesas se canaliza a opciones productivas, mientras el $48 \%$ se usa para el sostenimiento de las familias. ${ }^{50}$

$\mathrm{Al}$ incrementarse, el poder de compra de las familias migrantes provoca un aumento en los precios, de manera que familias que no reciben transferencias monetarias son desplazados de ciertos mercados,${ }^{51}$ lo que crea una forma de marginación completamente nueva en las comunidades afectadas. Comparado con los hogares que no tienen familiares en los EUA, los que reciben remesas aparentemente tienen un nivel de vida más alto. En estos hogares se pueden encontrar bienes de consumo que normalmente no se encuentran en las comunidades rurales, por ejemplo, juegos de cómputo, teléfonos inalámbricos, estéreos y máquinas de fax. ${ }^{52}$.

Los hogares de migrantes gastan una gran parte de sus ingresos fuera de los mercados locales y regionales; además, consumen más bienes importados. Por consecuencia, gran parte de las remesas se canalizan directamente hacia los centros urbanos y no

INEGI: www.inegi.gob.mx

Resto: $10 \%$ vivienda, $14 \%$ no se sabe, $14 \%$ no cabe en ninguna categoría, Durand el al, "Migradollars...", op. cil.

51 Douglas S. Massey \& Lawrence C. Basem, "Determinants of Savings, Remittances, and Spending Patterns among U.S. Migrants in four Mexican Communities", Sociological Inquiry, Vol. 62. No. 2, Spring, 1992, pp. 185207.

52 Durand \& Arias, op. cil. 
causan ningún beneficio en las economías locales. ${ }^{53} \mathrm{El}$ impacto de las transferencias monetarias en el desarrollo regional obviamente no sólo depende del monto, sino también del uso, es decir, del tipo de bienes adquiridos y de su integración económica con la región.

Trabajar por cuenta propia o en pequeñas empresas se considera la mejor manera de frenar la migración internacional en las regiones afectadas, porquie esto implica un desarrollo económico sostenible, basado en la región misma. ${ }^{54}$

Un desarrollo económico independiente es más probable si las inversiones juegan un papel importante en el uso de las remesas. Según Conway y Cohen, ${ }^{55}$ los siguientes usos se pueden considerar como inversiones: 1 . ahorros; 2 inversión en capital local (vivienda, terrenos); 3. inversión en capital humano (educación); 4. inversiones microeconómicas (empresas), y 5. sistemas para el soporte de la comunidad (infraestructura).

Por eso, el cuadro que determina la decisión para invertir ó consumir las remesas merece atención especial. Las personas que reciben remesas o "transferencias en bolso" deciden cómo utilizar el dinero, tomando en cuenta los deseos de los migrantes. Los recursos monetarios se pueden gastar inmediatamente o se pueden ahorrar para gastos en el futuro, para el consumo diferido y la inversión. La probabilidad de inversiones productivas se incrementa con la disponibilidad de recursos productivos en las regiones de origen, como lo son la educación, la habilidad de miembros de la familia para trabajar, la posesión de terrenos, vivienda privada o un negocio ya existente. Otra razón para in-

53 Richard C. Jones, "Remittances and Inequality;: A Question of Migration Stage and Ceographic Scale", Economic Geography, 74, 1998, pp. 8-25.

51. Agustín Escobar Latapí \& María de la O. Martínez Castellanos, "SmallScale Industry and International Migration in Guadalajara, México", Series on Development and International Migration in Mexico, Central America and the Caribbean basin, Volume IV, Ed. Diaz-Briquets, Sergio; Weintraub, Sidney, 1991, pp. 135-173.

55 Conway \& Cohen, op. cil. 
vertir las transferencias monetarias es un trabajo bien pagado y seguro en los EUA, ${ }^{56}$ que facilite al migrante hacer planes para el futuro y no implica la necesidad de consumo inmediato. De los recursos ganados, el porcentaje del dinero canalizado a inversiones también es más alto si el migrante cuenta con estatus legal. ${ }^{57}$

Muchos migrantes hacen sus viajes persiguiendo una cierta meta económica. Para ellos, trabajar en los EUA es la base del establecimiento de un nuevo negocio o una inversión planeada, en un negocio ya existente (la llamada "teoría del ingreso con meta" $\left.{ }^{2}\right)$. Muchas veces, las inversiones en las familias migrantes son escasas, interviene el hecho de que hay una ausencia de oportunidades para inversiones productivas en las regiones de origen. Los migrantes que trabajan y ahorran para realizar una cierta meta en promedio se quedan más tiempo en los EUA; como quieren realizar una cierta meta, también la probabilidad de que se vuelvan migrantes establecidos es más alta. ${ }^{59}$ En este contexto, tenemos que distinguir entre remesas y ahorros. Mientras las remesas nada más se usan en un $3.5 \%$ para fin los ahorros que se invierte es de $17 \%$. Este hecho también respalda la reflexión de Lindstrom.

Escobar Latapí/ Martínez Castellanos ${ }^{60}$ y Massey/ Parrado ${ }^{61}$ muestran el vínculo entre migración, remesas e inversiones en pequeñas industrias. Estos autores llegan a la conclusión de que la inversión en una industria pequeña ó mediana depende significativamente de las remesas y su función como una fuente de actividades empresariales. Sin embargo, hay que tomar en cuen-

56 Durand et al.,"Miradollars..."op. cit.

57 Massey \& Basem, op. cit.

58 Lijndstrom, op. cil.

59 Massey \& Basem, op. cit.

60 Escobar Latapí \& Martínez Castellanos, op. cil.

61 Massey, Douglas \& Parrado, "Migración y pequeña empresa", Ciudades, 35, julio-septiembre, 1997, pp. 34-40. 
ta que estos estudios sólo cubrieron comunidades urbanas. Massey \& Parrado ${ }^{62}$ encontraron que el $2 \%$ de los migrantes del grupo examinado establecieron pequeños negocios y $21 \%$ de todos los negocios fueron establecidos con la ayuda de ganancias de migrantes. En comparación con los migrantes rurales, la probabilidad de formar un negocio pequeño es más alta en los inversionistas urbanos con cierta educación. La mayoría de los negocios se pueden clasificar como comercio de menudeo $(4.9 \%)$ ó mayoreo (13\%), mientras las empresas productivas nada más llegan al 15\% y ningún negocio se estableció en el área de la agricultura. Estos hallazgos subrayan el hecho de que las inversiones en comunidades rurales y en el sector de la agricultura son muy bajas, por las escasas oportunidades de inversión. Aquí es muy importante tomar en cuenta que la mayoría de los migrantes proviene de comunidades rurales. También hay que ver que los estudios existentes no toman en cuenta los posibles efectos de "crowding out" de negocios establecidos con dinero ganado en los EUA. Por ejemplo, una tienda ya establecida quiebra por la competencia de otros que se establecen y se mantienen con la ayuda de las remesas. Además, es importante ver que las actividades de comercio al mayoreo y menudeo en las regiones con alta migración dependen del poder de adquisición de la comunidad; considerando que esto depende mucho de las remesas, podemos concluir que ningún negocio en estas regiones podría existir sin la ayuda de los dólares.

Las redes sociales existentes también son causa para actividades de inversión. Los clubes de los migrantes en las regiones de destino ahorran dinero para inversiones en sus comunidades de origen. ${ }^{63}$ Por ejemplo, los clubes de la comunidad de San Mateo

62 Douglas S. Massey \& Emilio A. Parrado, "International Migration and Business Formation in Mexieo", Sociological Quarlerly, Vol. 79, Numb. 1, March, 1998, pp. 1- 20.

(.3) Moctezuma, op. cil. 
en el municipio de Valparaíso, ahorraron dinero para pavimentar la carretera hacia la comunidad, iluminar las calles y preparar terrenos para cultivos.

A causa de la crisis en los años 80 se firmó el Convenio Único de Desarrollo, que contenía la participación de las comunidades beneficiadas con las inversiones federales en infraestructura. En Zacatecas, siendo el estado con la tasa más elevada de migración y el que recibe el mayor monto de dólar ss desde los EuA por habitante, además de ser uno de los estados más pobres de la República, se trató de canalizar parte de las remesas a la construcción de obras de infraestructura. Las organizaciones zacatecanas en los EUA mostraron interés en mantener vivos los lazos con su tierra natal y aportar recursos para el desarrollo de las comunidades. ${ }^{64}$ Pero fue hasta 1993, con el inicio del Programa 2 x 1, que se logró el aumento de las inversiones comunitarias en los municipios. Los clubes zacatecanos aportaron una tercera parte de las inversiones, el resto lo proporcionaron los gobiernos federal y estatal. Entre 1993 y 1997, en los municipios del estado de Zacatecas, se invirtieron un total de 33.4 millones de pesos con este programa. ${ }^{65}$ Durante el gobierno de Ricardo Monreal el programa 2 x 1 se ha mantenido bajo la denominación de programa 3 x 1. Con este concepto también se incorpora una aportación del municipio donde se realiza la obra. En el primer año de este programa se realizaron 93 obras públicas en 27 municipios, con una inversión de poco más de 48 millones de pesos; ${ }^{66}$ con el programa se realizaron obras como: pavimentación de carreteras, perforación de pozos, establecimiento de centros de computo, extensión de la red eléctrica, reparación y renovación de edificios públicos, etcétera.

64 Jesús Flores Olague, el al., Breve historia de Zacatecas, México, FCE, 1996.

65 Moctezuma, op. cil.

66 Folleto del Estado Zacatecas, 1999.

$\begin{array}{llllllllllllll}60 & \text { C } & \text { A } & \text { L } & \text { E } & \text { I } & \text { D } & \text { O } & \text { S } & \text { C } & \text { O } & \text { P } & \text { I } & \text { O }\end{array}$ 
Considerando una encuesta que abarca a 22 municipios Massey \& Parrado ${ }^{67}$ calculan que de los 2 mil millones de dólares que llegaron a México en forma de remesas en 1990, nada más 85 millones se usaron para inversiones. Sin embargo, hay que considerar lo siguiente: 1 . no sólo las inversiones tienen implicaciones en el crecimiento económico; 2. el consumo también aumenta la demanda de bienes y servicios; 3. esto aumenta los ingresos en los sectores de comercio y de la producción, y 4. parte de este impulso económico se transmite a los proveedores.

Estos efectos multiplicadores no sólo aumentan los ingresos de las familias migrantes, sino que también benefician a otros grupos sociales y mejoran la situación económica a nivel local, regional y nacional. El efecto total depende del grado en que la economía local o regional puede satisfacer los nuevos patrones de demanda presentadas por las familias de los migrantes. El impulso hacia la economía regional depende de que la demanda adicional pueda ser cubierta por la economía local. Considerando lo anterior, señalaremos que se ha visto que con el aumento del ingreso los patrones de consumo están sometidos a cambios; sería el caso de los bienes tradicionales -que se producen en la misma región-, que muchas veces son sustituidos por bienes importados. ${ }^{68}$ En este contexto es muy interesante ver que los migrantes gastan una gran parte de las remesas en la construcción de casas; sin embargo, la industria constructora de Zacatecas no ha mostrado un crecimiento significativo durante los últimos años. En otras palabras, el estado de Zacatecas importa capacidades de la industria constructora de los estado vecinos.

67 Douglas S. Massey, Emilio A. Parrado, "Migradollars: The remittances and savings of Mexican migrants to the USA", Population Research and Policy Review, 13, 1994, pp. 3-30.

68 Marta Guidi, Estigma y prestigio: la tradición de migrar en San Juan Mixtepec, Oaxaca, México, Bonn, Holos Verlag, 1992; Durand \& Arias, op. cil. 
Usando una matriz de contabilidad social se calcula que los 2 billones de dólares de remesas en 1990, crearon efectos multiplicadores e ingresos adicionales en la República Mexicana por 5.8 billones de dólares. ${ }^{69}$ Estos efectos indirectos también sirvieron para reducir el desequilibrio de ingresos dentro de los municipios, ya que las personas que no tienen familiares migrantes pueden participar del aumento en la actividad económica. ${ }^{70}$

\section{RESUMEN Y CONCLUSIÓN}

Hemos visto que la migración no sólo tiene impactos en el país de destino, sino también en los países de origen de los migrantes. Por eso, las políticas migratorias no sólo deberían fijarse en el país que importa fuerza de trabajo, sino también en el que la remite.

La migración de fuerza de trabajo en México es un fenómeno que se acentúa más en las áreas rurales y por sus raíces históricas es muy heterogénea en todo el país. Las redes sociales toman un papel muy importante en la organización de los flujos migratorios y de inversiones en las comunidades de origen. El libre comercio dará muchas oportunidades de trabajo, pero los impactos del TLCAN van a aumentar en el corto plazo los movimientos migratorios, ya que los migrantes potenciales no tienen fácil acceso a los trabajos industriales. Asimismo, el cambio de los patrones migratorios tiende a reducir los lazos entre las comunidades de origen y los migrantes establecidos en los EUA, lo que

69 Durand el al. "International Migration...", op. cil.

70 J. Edward Taylor \& T. J. Wyatt, "The Shadow Value of Migrant Remittances, Income and Inequality in a Household-farm economy", Journal of Development Studies, 32. No. 6, August, 1996, pp. 899-912; J. Edward Taylor, "Remittances and Inequality Reconsidered: Direct, Indirect and Intertemporal Effects", Journal of Policy Modeling, 14 (2), 1992, pp. 187 208. 
provocaría que en un futuro próximo el monto de dinero enviado podría también reducirse.

Hay algo claro: no se pude dudar que las remesas tienen un impacto importante en el desarrollo económico de las regiones expulsoras de migrantes, ni que ayudan a asegurar el mantenimiento de la familia, permiten un estándar de vida más alto y parte de ellas pueden ser invertidas en negocios pequeños o medianos. Sin embargo, gran parte de las transferencias monetarias no se usan de un modo productivo directo, porque se emplean para las necesidades indispensables de las familias. En las zonas rurales marginadas, la carencia de oportunidades para las inversiones productivas condiciona las posibilidades del crecimiento económico. Lo que se observa en estas regiones puede ser descrito más bien de un crecimiento económico que de un desarrollo. Los negocios que se establecen son de comercios al mayoreo o menudeo; aunque den empleo y aumenten los ingresos de la región, siguen dependiendo de los dólares que llega de los EuA. Por otro lado, las remesas que llegan a zonas urbanas se usan en una proporción más alta para fines productivos, lo que ayuda a que el desarrollo económico en las zonas urbanas sea mayor, respecto a las áreas rurales.

Hemos visto que las inversiones no sólo dependen de las circunstancias en México, sino también de la situación del migrante en los EUA. Un empleo seguro da más iniciativa para invertir que una situación inestable. Por un lado, la existencia de un negocio reduce la probabilidad de migrar, y por el otro, aumenta la probabilidad de invertir las remesas. Así, las familias que poseen un negocio no dependen tanto de la migración. Si un miembro de la familia decide irse "al otro lado", las ganancias se invierten en el negocio ya existente. Asimismo, la posesión de terrenos aumenta la probabilidad de invertir las ganancias en el lugar de origen. Por otro lado, parece que el dinero ganado en los EuA y que es invertido en el sector agrícola no produce ganancias suficientes para detener la migración, 
Es conveniente considerar que el desarrollo regional no sólo depende de la parte de remesas que se canaliza a inversiones productivas, sino también de la parte que se usa para adquirir bienes o servicios de la producción local. Este dinero tiene efectos multiplicadores dentro de la economía, porque así las empresas regionales participan de la demanda incrementada. Las pocas inversiones de los migrantes pueden ser explicadas por esta misma razón, como fue el fracaso de la industria maquiladora en Zacatecas. Gracias a las redes sociales, los costos de la migración son más bajos que los ingresos que se esperan en los EUA. Adicionalmente, éstos son mucho más altos de lo que se pueden ganar con un negocio propio en las comunidades de origen. Por eso, establecer un negocio para los migrantes no representa una alternativa viable, pero si representa una opción para las familias no-migrantes.

Finalmente, considero que es importante que se realicen otras investigaciones para encontrar la forma de incidir productivamente en la estructura económica regional de las áreas con alta migración, tratando de prevenir el síntoma de la "enfermedad holandesa". Sólo así se puede comenzar a avanzar en el crecimiento regional de las zonas rurales, apoyando las inversiones en la producción de bienes comerciables, que tradicionalmente se importan de las zonas urbanas. El programa $3 \times 1$ del gobierno del estado de Zacatecas puede tomar un papel importante en este renglón. Resta señalar la necesidad de capacitar a los habitantes de las comunidades para poder usar los potenciales de crecimiento económico existentes, no sólo dentro de la región, sino también los que resultan del libre comercio entre los EuA, Canadá y México. Hacen falta más investigaciones que identifiquen las restricciones que condicionan el desarrollo y la inversión, así como los factores que restringen el despegue económico de las zonas rurales. En esto consisten los desafíos que habremos de enfrentar. 
